\title{
Immunité innée - Le ciblage de la chromatine de la cellule hôte par les bactéries : quelles implications dans la réponse immunitaire innée ?
}

\author{
Innate immunity - Targeting of host cell chromatin by bacteria: \\ what are the implications for innate immune response?
}

\author{
L. Arbibe \\ C SRLF et Springer-Verlag France 2010
}

Les pathogènes bactériens ont développé diverses stratégies pour échapper au système immunitaire. L'identification récente de facteurs de virulence bactériens capables de se localiser dans le noyau de la cellule hôte et de modifier la structure de la chromatine indique que les bactéries manipulent la structure de la chromatine au décours de l'infection. La compréhension des mécanismes régulant ce nouvel aspect de l'interaction hôte-pathogène est essentielle pour identifier leurs implications fonctionnelles dans la régulation de la réponse immunitaire. Notre travail a permis de montrer qu'un pathogène bactérien tel que Shigella flexneri est capable de moduler la transcription de la cellule hôte en injectant une protéine de virulence appelée OspF dans le noyau de la cellule hôte [1]. OspF est une enzyme inactivant les MAPK-kinases Erk et p38. OspF est recruté au niveau $\mathrm{du}$ site promoteur d'un groupe spécifique de gènes de l'immunité innée et réprime la transcription de ces gènes en bloquant le recrutement de la machinerie transcriptionnelle. En fait, l'activité enzymatique d'OspF perturbe les niveaux de phosphorylation de l'histone $\mathrm{H} 3$ contactant le promoteur et rend la structure du nucléosome inaccessible au recrutement du facteur de transcription NF- $\mathrm{KB}$ et de la polymérase II. Ainsi, le pathogène S. flexneri «reprogramme » la réponse transcriptionnelle de la cellule épithéliale de façon précise en modulant l'information épigénétique sur des gènes clés de la réponse immunitaire innée. La production d'interleukine 8 joue un rôle central dans la physiopathologie de la shigellose, car elle contrôle l'afflux muqueux des polymorphonucléaires neutrophiles (PMN) et leur migration transépithéliale, conduisant ainsi à la rupture de l'intégrité de la barrière épithéliale nécessaire à l'invasion bactérienne. Nous avons donc analysé in vivo l'impact de OspF dans un modèle d'anse ligaturée de lapin. OspF réprime l'afflux de PNN dans la muqueuse et la lumière intestinale. Ce résultat corrèle donc avec les données transcriptionnelles obtenues in vitro et suggère une stratégie habile de survie bactérienne à la surface des muqueuses en réprimant la réponse immunitaire mucosale.

La cellule épithéliale est soumise à des stimuli potentiellement impliqués dans le dommage de l'ADN tels que le stress oxydant au décours de l'inflammation ou l'injection de toxines bactériennes capables d'induire des cassures doubles brins de l'ADN. Une seconde étude nous a permis de montrer que Shigella induit des cassures de l'ADN. La signification fonctionnelle de ce stress génotoxique au décours de l'infection aiguë reste à déterminer. Ainsi, ces deux études montrent que les pathogènes bactériens ont acquis la possibilité de "manipuler " la structure de la chromatine de la cellule hôte.

Conflit d'intérêt : l'auteur déclare ne pas avoir de conflit d'intérêt.

\section{Références}

1. Arbibe L, Kim DW, Batsche E, et al (2007) An injected bacterial effector targets chromatin access for transcription factor NF-kappaB to alter transcription of host genes involved in immune responses. Nat Immunol 8(1): 47-56

L. Arbibe $(\square)$

Unité de pathogénie microbienne moléculaire,

Inserm U786, Institut Pasteur, 28, rue du Docteur-Roux,

F-75724 Paris cedex 15, France

e-mail : arbibe@pasteur.fr 